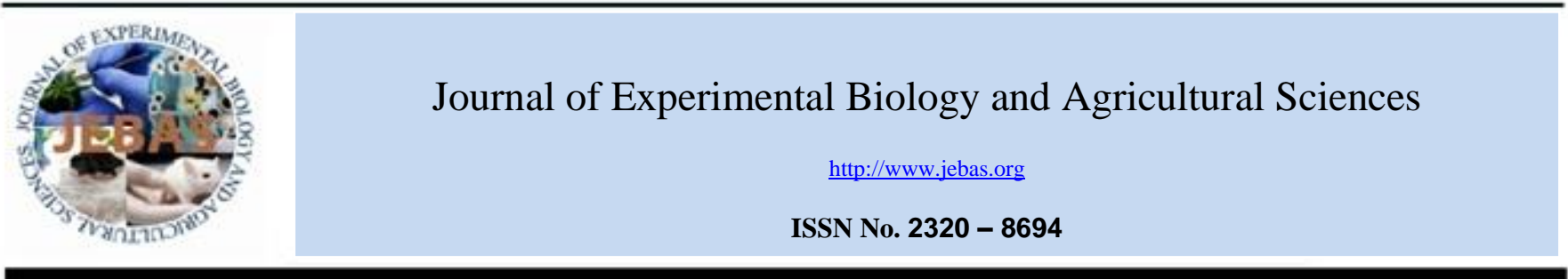

\title{
WHOLE GENOME SEQUENCING OF CLASSICAL SWINE FEVER VIRUS CIRCULATING IN INDIA AND ANALYSIS OF ANTIGENIC RELATION WITH C-STRAIN AND HCLV/INDIA
}

\author{
Neelam Tomar ${ }^{1}$, Veena Sharma ${ }^{2}$, Jeny K John ${ }^{1}$, Menaka Sethi ${ }^{1}$, Pradeep K. Ray ${ }^{3}$, \\ Rahul Singh Arya ${ }^{4}$, Tareni Das ${ }^{1}$, K. Dhama ${ }^{1}$, G. Saikumar ${ }^{1 *}$
}

${ }^{1}$ Division of Pathology, ICAR-Indian Veterinary Research Institute, Izatnagar-243122, Bareilly, Uttar Pradesh, India

${ }^{2}$ Department of Bioscience and Biotechnology, Banasthali University, Rajasthan-304022, India.

${ }^{3}$ ICAR Research complex for eastern region, Patna, Bihar-800014, India.

${ }^{4}$ Department of Veterinary Pathology, College of Veterinary sciences and Animal husbandry, Selesih, Aizawl-796015, Mizoram

Received - March 29, 2018; Revision - May 05, 2018; Accepted - June 15, 2018

Available Online - June 20, 2018

DOI: http://dx.doi.org/10.18006/2018.6(3).509.515

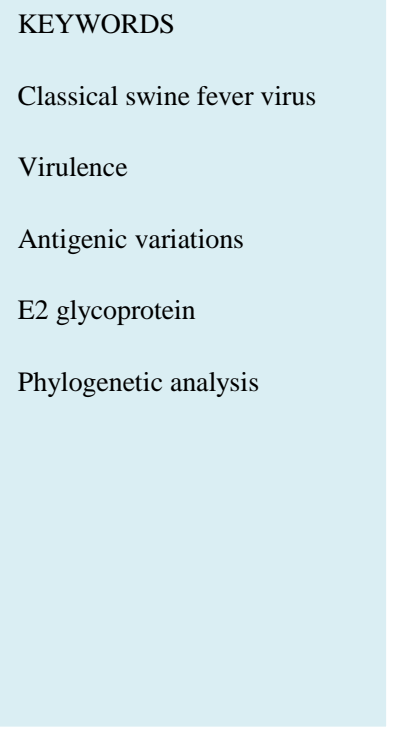

\begin{abstract}
Majority of live attenuated vaccine against classical swine fever virus (CSFV) including HCLV/India are derived from Chinese vaccine strain, $\mathrm{C}$-strain which is genogroup 1 virus. Presently genotype 2 viruses are predominantly circulating in Indian field. Therefore the present study was carried out to find out the efficacy/failure of ongoing vaccine by whole genome sequencing and analysis. The whole genome sequences of Indian classical swine fever virus isolate CSFV-UP-BD-SKN-11 (SKN-11) (Accession no. KC533776) was done in 11 overlapping fragments. SKN-11 composed of a 373 nucleotide (nt) 5'NTR, an 11,697-nt open reading frame (ORF) encoding a polyprotein of 3,898 amino acids (AAs), and a 227-nt 3'NTR with total length of genome 12297 nts. SKN-11 strain belongs to the genotype 2.2 which is dominantly circulating in Indian field. Based on amino acid sequence of B-cell epitopes, T-cell epitopes and conformational residues there was a remarkable antigenic variation between SKN-11 (genotype 2.2) and the currently using vaccine strains, HCLV/India and C-strain (genotype 1). Furthermore, AA at position L889Q showed an extremity change from hydrophilic to hydrophobic, which may alter the antigenicity and virulence of CSFV. Close antigenic relationship was also observed with Chinese strains especially Strain-39. The vaccination programs without genotype change can resulted in alterations in viral pathogenicity. These findings may contribute to further understanding the phylogeny and antigenic variation in CSFV and development of a more effective vaccine.
\end{abstract}

* Corresponding author

E-mail: saikumarivri@gmail.com (Dr. G. Saikumar)

Peer review under responsibility of Journal of Experimental Biology and Agricultural Sciences.

Production and Hosting by Horizon Publisher India [HPI] (http://www.horizonpublisherindia.in/).

All rights reserved.
All the article published by Journal of Experimental Biology and Agricultural Sciences is licensed under a Creative Commons Attribution-NonCommercial 4.0 International License Based on a work at www.jebas.org.

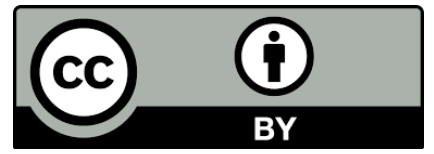




\section{Introduction}

Classical swine fever (CSF) is a notifiable disease of domestic pigs in India, Bhutan and Nepal (Monger, 2015) caused by classical swine fever virus (CSFV), a small (40-60 nm) enveloped virus with a single stranded RNA genome of positive polarity, glycosylated membrane proteins and an icosahedral nucleocapsid. The genome is of about 12,300 base pairs and comprises one large Open Reading Frame (ORF) between the 5' and 3' untranslated region (UTR) (Tautz et al., 2015). The ORF codes for a polyprotein of 3,898 AAs, which produce four structural proteins $\left(\mathrm{C}, \mathrm{E}^{\mathrm{rns}}, \mathrm{E} 1, \mathrm{E} 2\right)$ and eight non structural proteins $\left(\mathrm{N}^{\mathrm{pro}}, \mathrm{p} 7, \mathrm{NS} 2\right.$, NS3, NS4A, NS4B, NS5A and NS5B) on processing co- and post-translationally by both host and virus encoded proteases (Lin et al., 2014). CSFV comprises three different genotypes 1, 2, and 3 , with three to four subgenotypes each (Postel et al., 2013; Blome et al., 2015). Smaller fragment of $5^{\prime}$-NTR (150 nt), E2 (190 nt), and NS5B (409 nt) sequence of CSFV are widely used for phylogenetic analyses (Paton et al., 2000) but for high-resolution analyses towards the understanding of genetic variability and virus evolution, the full-length E2 gene sequence (1,119 nt) and full-genome sequences are more appropriate (Postel et al., 2012; Beer et al., 2015; Zhang et al., 2015). This strategy is recommended by the EU and OIE Reference Laboratory for CSF as it provides a solid and improved basis for CSFV molecular epidemiology.

All three viral glycoproteins (Erns, E1, and E2) have been associated with CSFV virulence (Risatti et al., 2005a; Risatti et al., 2005b). The E2 protein is the most important immunogen of $\mathrm{CSFV}$, and it stimulates the production of neutralizing antibodies that offer shield against deadly CSFV infection (Beer et al., 2015); it also plays important roles in the viral life cycle, and in the entry of the virus into host cells. Erns also called E1 lacks a typical membrane anchor and it is secreted from infected cells associated with mature virions. A study shows that the NS2, NS3, NS4A, NS4B, NS5A and NS5B are required for CSFV replication (Risager et al., 2013).

Majority of live-attenuated vaccine strains used in different countries belong to group 1 (Paton et al., 2000), including the subgroup 1.1 strain, HCLV/India and Chinese lapinized vaccine strain, C-strain which was derived by serial passage of a virulent strain in rabbits. The variation in proteins and antigenic structure of any virus may lead to alteration in its pathogenecity. Two independent studies reported that subgroup 2.1 strains recently branched away from the vaccine C-strain and became dominant in China (Chen, et al., 2008; Zhu, et al., 2009). In India, four genogroups 1.1, 1.2, 2.1 and 2.2 have been reported with the majority being genogroups 2.2 and 1.1. Despite rigorous vaccination, there has been an increased incidence of outbreaks caused by CSFV genogroup 2.2. Detailed genome information of genotype 2.2 field isolates from India is very less so it was decided to full genome sequencing of genotype 2.2 isolate in order to investigate the antigenic diversity between genotype 2.2 viruses circulating in Indian field and the vaccine strains, HCLVIndia and C-strain (genotype 1).

\section{Materials and Methods}

The sample was collected from CSFV outbreaks reported at an organized pig farm of Utter Pradesh, India. Total RNA was extracted from pooled homogenate of tissue; tonsil, spleen, lymph node and blood sample of infected pigs using Trizol reagent (Invitrogen) and cDNA was synthesized using SuperScriptTM III RT (Invitrogen). The whole genome of CSFV field sample (SKN11) was amplified in 11 fragments by PCR using overlapping primer pairs. The PCR conditions for different sets of primers were standardized and the amplified PCR products were sequenced using commercial facility. The sequences of different overlapping fragments of CSFV genome thus generated were identified by NCBI-BLAST program (http://blast.stva.ncbi.nlm.nih.gov/Blast.cgi) using existing CSFV sequence database. The complete genome sequence and individual genes/regions sequence were then aligned with respective available sequences of other CSFVs using MegAlign program of Lasergene software for annotation of full length sequence thus generated was submitted to NCBI-GenBank database.

Full genome sequences of CSFVs of were downloaded from NCBI database and used along with complete genome sequence of SKN-11 for phylogenetic analysis. The phylogenetic analysis was carried out using ClustalW program and MEGA 6.06 software package (Tamura et al., 2013). Only statistically significant bootstrap values $(\geq 70.0 \%)$ are indicated and Strains 94.4/IL/94/TWN was kept as out groups. Antigenic relation of SKN-11 with C-strain and HCLV/India was analyzed by comparing the AAs sequences of important $\mathrm{mAb}$ binding sites; conformational residues, B-cell epitopes, T-cell epitopes.

\section{Results}

The complete genome sequences of CSFV isolate SKN-11 composed of a 373-nt 5'NTR an 11,697-nt open reading frame (ORF) encoding a polyprotein of 3,898 AAs and a 227-nt 3'-NTR with total length of genome 12297nts. NCBI BLAST results of full genome nucleotide sequence of SKN-11 revealed homology 96\%-98\% with other isolates of genogroup 2.2 from India and 93\% with Chinese recombinant virus Strain-39. The isolate SKN11 showed 89\% homology with group 2.1 Indian isolate IND/AS/GHY/G4 and 90\% with other group 2.1 isolates such as Padarborn, Taiwanese isolate 96TD which was followed by some other genogroup 2.1 Chinese viruses (SXYL06, GXWZ02, HEBZ, YC11WB, PC11WB) and genogroup 2.3 viruses with 
homology $88 \%$. SKN-11 showed $85 \%$ identity with genogroup 1 viruses. At AA level isolate SKN-11 shared $99-97 \%$ homologous with other Indian isolates, CSFV-UP-GZ-NVD-11, CSFVUP-BR-KHG-06 and CSFV-UP-ND-169-11. Next highest $95 \%$ homology was found with Strain-39 and Bergen. The full genome sequence of the isolate SKN-11 was submitted to the Genebank and acquired accession number KC533776. BLAST result of full E2 protein revealed close homology (96-99\%) between Indian strains of group 2.2 such as Mathura, LAL-290, CSFV-UP-BD-BD-11, MIB56, CSFVUP-BR-793-09, and CSFV-UP-GZ-NG39-10. and Chinese virus Strain-39 (96\% homology). E2 region of SKN-11 showed 97\% homology with CSF 1059 and CSF1060 isolated in Nepal in 2011, 93\% homology with group 2.2 isolate CSF0073 from Austria in 1990, 90\% with Sukoharjo (Indonesia) isolated in 2007, 92\% with CSF0378 which was isolated in year 1994 from Czech Republic, 93\% homology with isolate 84KS1 which was isolated in 1995 from Taiwan, 92\% CSF0573 Parma isolated in 1998 in Italy, 90\% with CSF0014 Isolated from Germany.

3.1 Phylogenetic analysis on the basis of complete genome sequences

Phylogenetic analysis based on complete genome sequences of the available CSFV isolates was carried out along with Indian isolate reported in present study. Genetic variability or recombination in Strain 39 showed the evidence of distinct branch segregation between strain 39 and SKN-11, CSFV-UP-GZ-NVD-11 (NVD-11), CSFV-UP-ND-169-11(ND-169-11), CSFV-UPBR-KHG-06 (KHG-06), Lal-290. Isolate SKN11 fall under subgroup 2.2. SKN-11 clustered with earlier published Indian CSFV isolates NVD-11, ND-169-11, KHG-06 and Lal-290. Phylogenetic analysis conducted in this study confirms the presence of genotype 1 and 2 in India. Genotype 2.2 is dominantly circulating all over India (Figure 1).

\subsection{Analysis of E2 protein peptide structure}

The deduced peptide structure of new Indian isolates in comparison of reference isolates and vaccine strains was analyzed for AA replacements. Variation AA in B-cell epitope

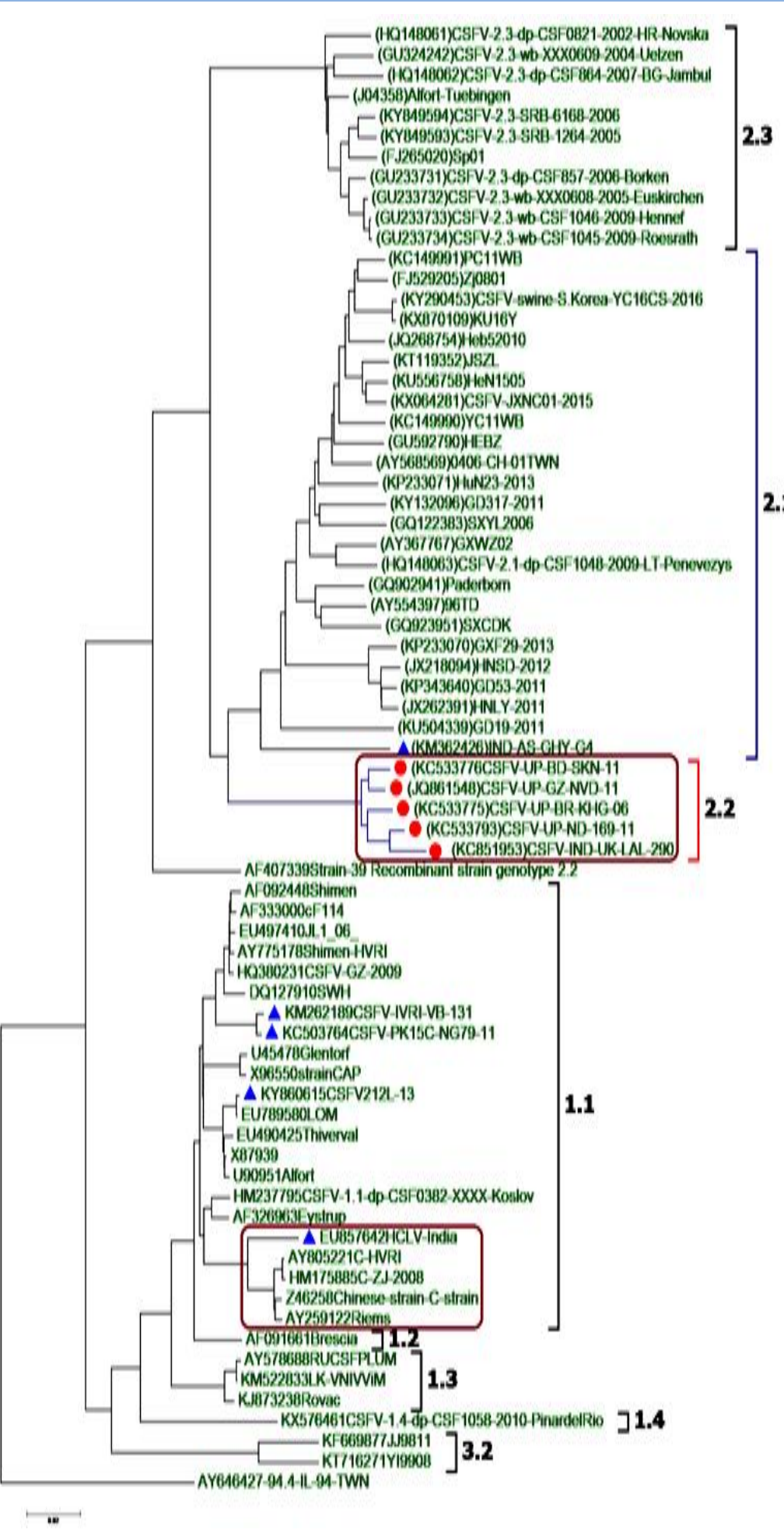

Figure 1 Phylogenetic tree based on the full genome sequence of Indian CSFV and reference sequences originating from GenBank (isolate name, accession number) were calculated by the Neighbor Joining method including bootstrap values for 1000 repetitions. Orange circle is showed genotype 2.2 and blue triangle showed genotype 2.1 and 1.1 isolates from India. 
spanning AA position 693-716, 712-728, 844-865 and T-cell epitopes spanning from 696-710, 690-714, 713-740, 736-750, $741-765,828-848,996-1010,1001-1015$ in E2 protein was noticed (Table 1). Variable AA was found at positions E706G and G713E. AA position D847E, V854M, M860T and N863K located in the neutralizing epitopes is variable among the $\mathrm{SKN}-11$ and Vaccine strains. At position 738 in HCLV/India and C-strain Valine is replaced by Isoleucine (hydrophobic) in SKN-11 and Strain-39. In T-cell epitopes AA position 761, Glutamic acid and Lysin is present in HCLV/India, C-strain but in SKN-11, Strain39, Arginine. In epitope spanning from AA 996-1010, position 1000 has glycine a hydrophobic AA present in HCLV/India while in SKN-11, aspartic acid a charged residue was found. In position 1002 which is also overlapped by B-cell epitope from position 1001-1015, AA cystine (polar residue) is present in HCLV/India which is replaced by tyrosine (polar aromatic residue) in SKN11.
At position 886/Threonine (polar AA) is present in vaccine strains (HCLV/India, C-strain), while in SKN and Strain-39, AA Isoleucine (hydrophobic) was present. At position 889/Leucine (hydrophobic) was present in HCLV/India and C-strain while SKN-11 and Srain-39 have Glutamine (polar). These Residues locally effect hydrophobicity and predicted beta-sheet conformation. Comparative analysis of AA important for monoclonal antibody binding showed that AA position $738 / \mathrm{V}$ (hydrophobic), 902/G in HCLV/India and the C- strain was replaced by $738 / \mathrm{I}$ (hydrophobic) and 902/E in SKN-11 and Strain-39.

\section{Discussion}

New Indian isolate $\mathrm{SKN}-11$ belonging to genotype 2.2 showed close homology (96\%-98\%) with other isolates of genogroup 2.2

Table 1 showed the details of comparative analysis based on amino acid sequence of B-cell epitopes, T-cell epitopes and conformational residues of isolate SKN-11 (genotype 2.2) and the vaccine strains, HCLV/India and C-strain (genotype 1).

\begin{tabular}{|c|c|c|c|}
\hline $\begin{array}{l}\text { AA in full } \\
\text { genome }\end{array}$ & $\begin{array}{l}\text { HCLV-India, C-strain (1.1) (Vaccine } \\
\text { strain) }\end{array}$ & SKN-11 (2.2) & $\begin{array}{l}\text { Strain-39 (China) } \\
(2.2)\end{array}$ \\
\hline \multicolumn{4}{|c|}{$\begin{array}{l}\text { Amino acid replacements in AA important for binding neutralizing antibodies of CSFV in B-cell epitope in E2 protein spanning from AA, } \\
693-716,712-728,844-865 \text {. }\end{array}$} \\
\hline 706 & Glutamic acid (charged) & Glycine (hydrophobic) & Glutamic acid(charged) \\
\hline 713 & Glycine (hydrophobic) & Glutamic acid (charged) & Glutamic acid (charged) \\
\hline 777 & Asparagine & Serine (polar) & Serine (polar) \\
\hline 847 & Aspartic acid (charged) & Glutamic acid (charged) & Glutamic acid (charged) \\
\hline 854 & Methionine (hydrophobic) & Valine (hydrophobic) & Valine (hydrophobic) \\
\hline 860 & Threonine (polar) & Methionine (hydrophobic) & Methionin (hydrophobic) \\
\hline 863 & Asparagine (polar) & Glutamine/Lysine(charge)d) & Lysine (charged) \\
\hline \multicolumn{4}{|c|}{ Variable aa positions in T-cell epitopes in E2 protein spanning from AA 736-750,713-740, 741-765, 828-848, 996-1010, 1001-1015. } \\
\hline 723 & Asparagine (polar) & Serine (polar) & Serine (polar) \\
\hline 725 & Aspartic acid (charged) & Glycine (hydrophobic) & Glycine(hydrophobic) \\
\hline 738 & Valine(Hydrophobic) & Isoleucine (hydrophobic & Isoleucine (hydrophobic) \\
\hline 761 & Glutamic acid /Lysine & Arginine (polar) & Arginine (polar) \\
\hline 1000 & Glycine/ Aspartic Acid & Aspartic acid (charged) & Aspartic Acid (charged) \\
\hline 1002 & Cysteine (non polar) & Tyrosine (polar) & Tyrosine (polar) \\
\hline \multicolumn{4}{|c|}{ Residues which locally effect hydrophobicity and predicted beta-sheet conformation } \\
\hline 886 & Threonine (polar) & Isoleucine (Hydrophobic) & Isoleucine (Hydrophobic) \\
\hline 889 & Leucine (hydorphobic) & Glutamine (polar) & Glutamine (polar) \\
\hline \multicolumn{4}{|c|}{ Variable amino acid important for binding monoclonal antibodies of CSFV } \\
\hline 738 & Valine (hydrophobic) & Isoleucine (Hydrophobic) & Isoleucine (Hydrophobic) \\
\hline 902 & Glycine (hydrophobic) & Glutamic acid (charged) & Glutamic acid (charged) \\
\hline
\end{tabular}

Journal of Experimental Biology and Agricultural Sciences http://www.jebas.org 
viruses from India and $93 \%$ with Chinese recombinant virus Strain-39, a recombinant CSFV from China (He et al., 2007).

The virulence of Strain-39 strain cannot be classified into a particular genotype because the 5'NTR, 3'NTR and the NS5A/B genes are similar to genotype 1.1 isolates, the structural genes are homologous to genotype 2.1 strains while, E2 gene based tree grouped into subgroup 2.2. Moreover, the original sequence of the Strain-39 is not known because the sequence analysis was done after the $32^{\text {nd }}$ passage in cell culture. Chen \& Chen, (2014) challenged previous study (He et al., 2007) and concluded that CSFV might have relatively high frequency of homologous recombination events. BLAST result of full E2 indicating some clue regarding origin and spreading of recent Indian isolate as it showed the close relationship with other CSFVs from countries sharing border area with India such as Nepal and China. SKN-11 fall under genogroup 2.2 clustered with Strain-39 and other previously reported Indian isolates. Phylogenetic analysis segregated virulent, moderately virulent and low virulent or vaccine strains into distinct clusters. The majority of the virulent and vaccine strains belonged to the genotype 1 while; moderately virulent strains grouped into genotype 2 and 3. Typical historical strains includes all live-attenuated vaccine strains, comes under group 1, whereas the dominantly circulating strains belongs to gnogroup 2. However, the isolates circulating in Taiwan and some other regions belong to group 3 (Huang et al., 2014).

The E2 protein is the main immunogen of CSFV, and it induces the production of neutralizing antibodies that provide protection against lethal challenge and it also plays multiple roles in the viral life cycle (Beer et al., 2015). The genetic variability with vaccine strain has also been studied in the past by different workers. A number of AAs in E2 are subject to intensive selection in nature for better survivability of virus against the adapting host immune system. These positions have been recognized in monoclonal antibody binding sites (Chen et al., 2008).

The B-cell epitopes induces CSFV neutralizing antibodies (Dong $\&$ Chen, 2006) while the T-cell epitope was accounted for to be a powerful $\mathrm{T}$ cell antigen in vitro equipped for initiating lymphoproliferation, IFN-gamma and effector CTL reactions (Armengol et al., 2002). Dong \& Chen, (2006) have studied a peptide vaccine based on a B-cell epitope (AA 844-865) of Cstrain in pigs and found that the vaccine was able to induce neutralizing antibodies and protect the challenge of virulent Shimen strain of the same subgroup 1.1. But, Chen et al. (2008) pointed the uncertainty for efficiency of using genotype 1 vaccine strain in providing cross-protection against Group 2 viruses. This is already being observed that group-2 strains such as GXWZ02, HEBZ09, SXCDK09, SXYL06 and ZJ0801 have caused many outbreaks in China in spite of rigorous vaccination. In new Indian isolate SKN-11and most of group 2 viruses at AA position 777, Asparagine was replaced with Serine. Asparagine is unique for HCLV/Indian, C-strain and group 1 isolates. Replacements of AA residues D725G, N777S, D847E, M854V, T860M and N863K were noticed in CSFV isolate SKN-11 similar to previous studies (Chen et al., 2010a). The moderate increase in binding efficiency was observed with these replacements. The residue N777 is indispensable for the specifity of $\mathrm{mAb}$. This epitope is present only in group 1 and absent in group 2 CSFVs (Tong et al., 2015). The significant variations were observed between Indian isolate SKN-11 (2.2) and vaccine strains in terms of B-cell and T-cell epitope analyses. The stimulating peptides from 996 to 1010 and 1001 to 1015 of the E2 region are part of a B-cell epitope. Importantly AA position 844-865, variability was found in SKN11 as it is most highly conserved among different strains of CSFV (Dong \& Chen 2006). Three important AA substitutions identified by Chen et al. (2010a) at AA position 713-E, 723-R/S, and $779-\mathrm{V}$ were found variable in isolate $\mathrm{SKN}-11$ as compared to the vaccine strains. Chen et al. (2010) demonstrated that Single AA substitutions of G713E, N723S, and S779A responsible for the antigenic variation between the C-strain and subgroup 2.1 strains. Notably, a G713E substitution caused the most dramatic enhancement of binding of the variant $\mathrm{C}$-strain $\mathrm{E} 2$ protein to anti CSFV serum. AA 713E was critical for antigenic specificity of field strain (94.4/IL/94/TWN). Single AA change between a group 1 vaccine strain LPC and a group 3 field isolate could totally reverse the mAbs binding pattern (Chang et al., 2010). AA position in E2 protein D723S, N725G, T738 V, G761R and S777N replacements were observed in sites under positive pressure selection might have been involved in the viral escape to the neutralizing antibodies (Pérez et al., 2012). The G761R mutation can alter the virulence of the virus inducing variation on the clinical manifestations of the disease in the field. Chang et al. (2012) demonstrated the residue E-902 at the C-terminal region were critical for specific binding to mAbs. CSFV isolate SKN11 has variable AA with vaccine strains, HCLV-India,C-strain at residues 886 and 889 , which locally effect hydrophobicity and predicted beta-sheet conformation (Resatti et al., 2005a). In this investigation, we noticed an extremity difference of AA L889Q from hydrophilic to hydrophobic, which may add to a difference in the antigenicity and virulence of CSFV (Hu et al., 2016).

Chen et al. (2010b) reported that single mutations in the E2 B/C domains could result into variations in viral neutralization. Further, Hu et al. (2016) recognized the exclusive molecular characteristics of the $2.1 \mathrm{~d}$ isolates which can help to the adaptive advancement of CSFV under $\mathrm{C}$-strain vaccination. Taken together, variability in AAs within antigenic units may result in the antigenic variation. 


\section{Conclusion}

The results indicate that the circulating CSFV strains SKN-11 in Indian field was clustered in subgenotype 2.2. Majority of CSFV live attenuated vaccine are derived from Chinese vaccine strain C-strain that belongs to group 1 virus. Presently genotype 2 viruses are predominantly circulating in India. The AA replacement analysis of some important B-cell epitopes, T-cell epitopes and conformational residues showed that there were striking differences between CSFV isolate $\mathrm{SKN}-11$ group-2 virus and the vaccine strains (HCLV/India and C-strain). Furthermore AA at position L889Q showed an extremity change from hydrophilic to hydrophobic, which may transform the antigenicity and virulence of CSFV. It also revealed the close antigenic relationship with Chinese strains especially Strain-39. The moderate virulence nature of the CSFV genogroup 2, and vaccine failure could result in mild clinical and subclinical disease and this could also result in prolonged viral shedding into the environment. The new genome sequences may contribute to further understanding the phylogeny and antigenic variation in CSFV and development of a more effective vaccine.

\section{Acknowledgement}

The authors are highly thankful for the Indian Veterinary Research Institute for providing facilities to carry out the research work at IVRI, Izatnagar.

\section{Conflict of Interest}

The authors declare that there is no conflict of interest regarding the publication of this research paper.

\section{References}

Armengol E, Wiesmüller KH, Wienhold D, Büttner M, Pfaff E, Jung G, Saalmüller A (2002) Identification of T-cell epitopes in the structural and non-structural proteins of classical swine fever virus. Journal of general virology 83: 551-560.

Beer M, Goller KV, Staubach C, Blome S (2015) Genetic variability and distribution of Classical swine fever virus. Animal Health Research Reviews 16: 33-39.

Blome S, Staubach C, Henke J, Carlson J, Beer M (2015) Genetic variability and distribution of Classical swine fever virus. Animal Health Research Reviews 16: 33-39.

Chang CY, Huang CC, Deng MC, Huang YL, Lin YJ, Liu HM, Lin YL, Wang FI (2012) Antigenic mimicking with cysteinebased cyclized peptides reveals a previously unknown antigenic determinant on E2 glycoprotein of classical swine fever virus. Virus Research 163: 190-196.
Chang CY, Huang CC, Lin YJ, Deng MC, Chen HC, Tsai CH, Wang FI (2010) Antigenic domains analysis of classical swine fever virus E2 glycoprotein by mutagenesis and conformationdependent monoclonal antibodies. Virus Research 149: 183-189.

Chen N, Hu H, Zhang Z, Shuai J, Jiang L, Fang W (2008) Genetic diversity of the envelope glycoprotein E2 of classical swine fever virus: recent isolates branched away from historical and vaccine strains. Veterinary Microbiology 127: 286-299.

Chen N, Li D, Yuan X, Li X, Hu H, Zhu B (2010a) Genetic characterization of E2 gene of classical swine fever virus by restriction fragment length polymorphism and phylogenetic analysis. Virus Genes 40: 389-396. doi: 10.1007/s11262-0100465-8.

Chen N, Tong C, Li D, Wan J, Yuan X, Li X (2010b) Antigenic analysis of classical swine fever virus E2 glycoprotein using pig antibodies identifies residues contributing to antigenic variation of the vaccine $\mathrm{C}$-strain and group 2 strains circulating in China. Virology Journal 7: 378.

Chen Y, Chen YF (2014) Extensive homologous recombination in classical swine fever virus: A re-evaluation of homologous recombination events in the strain AF407339. Saudi Journal of Biological Sciences 21: 311-316.

Dong XN, Chen YH (2006) Spying the neutralizing epitopes on E2 N-terminal by candidate epitope-vaccines against classical swine fever virus. Vaccine 24: 4029-4034.

He CQ, Ding NZ, Chen JG, Li YL (2007) Evidence of natural recombination in classical swine fever virus. Virus Research 126 : 179-185.

Hu D, Lv L, Gu J, Chen T, Xiao Y, Liu S (2016) Genetic diversity and positive selection analysis of classical swine fever virus envelope protein gene E2 in east China under C-strain vaccination. Frontiers in Microbiology 7: 85.

Huang YL, Deng MC, Wang FI, Huang CC, Chang CY (2014) The challenges of classical swine fever control: modified live and E2 subunit vaccines. Virus Research 179: 1-11.

Lin Z, Liang W, Kang K, Li H, Cao Z, Zhang Y (2014) Classical swine fever virus and $\mathrm{p} 7$ protein induce secretion of IL-1 $\beta$ in macrophages. Journal of General Virology 95: 2693-2699.

Monger VR (2015) Epidemiology of selected pig viral diseases in Bhutan. PhD thesis submitted to the Utrecht University, Utrecht, Netherlands. 
Paton DJ, McGoldrick A, Greiser-Wilke I, Parchariyanon S, Song JY, Liou PP, Belak S (2000) Genetic typing of classical swine fever virus. Veterinary Microbiology 73: 137-157.

Pérez LJ, de Arce HD, Perera CL, Rosell R, Frías MT, Percedo MI, Tarradas, J, Dominguez P, Núñez JI, Ganges L (2012) Positive selection pressure on the $\mathrm{B} / \mathrm{C}$ domains of the $\mathrm{E} 2$-gene of classical swine fever virus in endemic areas under $\mathrm{C}$-strain vaccination. Infection, Genetics and Evolution 12: 1405-1412.

Postel A, Schmeiser S, Bernau J, Meindl-Boehmer A, Pridotkas G, Dirbakova Z, Becher P (2012) Improved strategy for phylogenetic analysis of classical swine fever virus based on fulllength E2 encoding sequences. Veterinary Research 43: 50.

Postel A, Schmeiser S, Perera CL, Rodríguez LJP, FriasLepoureau MT, Becher P (2013) Classical swine fever virus isolates from Cuba form a new subgenotype 1.4. Veterinary Microbiology 161: 334-338.

Risager PC, Fahnøe U, Gullberg M, Rasmussen TB, Belsham GJ (2013) Analysis of classical swine fever virus RNA replication determinants using replicons. Journal of General Virology 94: 1739-1748.

Risatti GR, Holinka LG, Lu Z, Kutish GF, Tulman ER, French RA, Borca MV (2005b) Mutation of E1 glycoprotein of classical swine fever virus affects viral virulence in swine. Virology 343 : 116-127.

Risatti GR, Borca MV, Kutish GF, Lu Z, Holinka LG, French RA, Tulman ER, Rock DL (2005a) The E2 glycoprotein of classical swine fever virus is a virulence determinant in swine. Journal of Virology 79: 3787-3796.

Tamura K, Stecher G, Peterson D, Filipski A, Kumar S (2013) MEGA6: molecular evolutionary genetics analysis version 6.0. Molecular Biology and Evolution 30: 2725-2729.

Tautz N, Tews BA, Meyers G (2015) The molecular biology of pestiviruses. Advances in Virus Research 93: 47-160.

Tong C, Chen N, Liao X, Xie W, Li D, Li X, Fang W (2015) The epitope recognized by monoclonal antibody $2 \mathrm{~B} 6$ in the $\mathrm{B} / \mathrm{C}$ domains of classical swine fever virus glycoprotein E2 affects viral binding to hyperimmune sera and replication. Journal of Microbiology and Biotechnology 25: 537-546.

Zhang H, Leng C, Feng L, Zhai H, Chen J, Liu C, Kan Y. (2015) A new subgenotype $2.1 \mathrm{~d}$ isolates of classical swine fever virus in China, 2014. Infection, Genetics and Evolution 34: 94-105.

Zhu Y, Shi Z, Drew TW, Wang Q, Qiu H, Guo H, Tu C. (2009) Antigenic differentiation of classical swine fever viruses in China by monoclonal antibodies. Virus Research 142: 169-174. 